\section{Optimización de la conservación de guayaba (Psidium guajava) en ensilado líquido mediante metodología superficie-respuesta}

\section{Optimization of guava conserva- tion (Psidium guajava) in liquid silage through surface-response methodology}

\author{
Vicente Castañeda', Polito Huayama ${ }^{2}$ y Jonathan Campos ${ }^{3}$
}

\section{RESUIMEn}

En la presente investigación, se estudió la optimización de la conservación de guayaba (Psidium guajava) en ensilado líquido mediante metodología superficie-respuesta, en función de las variables: estado de madurez de la guayaba, concentración de cachaza y tiempo de fermentación. La variable respuesta fue la mayor actividad antioxidante equivalente a vitamina C (mg/100 g), durante la producción de ensilado líquido de guayaba, determinado mediante espectrofotometría. Los valores de las variables evaluadas fueron: estado de madurez (maduro, pintón y verde), cachaza (250, 200 y $150 \mathrm{ml})$ y 15, 25 y 35 días de fermentación. Se empleó el diseño experimental de Box-Behnken y se predijo una concentración máxima de (ATa) antocianos totales de 3,72 mg/100 g y se aplicó el ABTS (método para determinar la actividad antioxidante) $133 \mathrm{mg} / 100 \mathrm{~g}$ de VCEAC (actividad antioxidante equivalente a vitamina $\mathrm{C}$ ). Los valores de las variables fueron: frutos maduros de guayaba, $250 \mathrm{~mL}$ de cachaza diluida en 1 litro de agua de mesa y 35 días de fermentación. A los resultados de aplicar el diseño de Box-Behnken, se les aplicó el análisis de regresión múltiple para determinar los coeficientes de las ecuaciones polinomiales, para, con ella, obtener líneas de superficie de respuesta para la determinación de la actividad antioxidante equivalente a vitamina $\mathrm{C}$. Todos estos cálculos y gráficos estadísticos fueron realizados en el software Statgraphics v. 5.1. Con estos valores óptimos se elaboró el ensilado líquido de guayaba, que fue sometida a análisis fisicoquímicos, análisis espectrofotométrico y evaluación organoléptica. Las pruebas dieron resultados satisfactorios para el uso en el suplemento alimenticio de animales menores.

\section{PALRBRAS ClaVE}

Optimización · ensilado · líquido · guayaba

\section{INTRODUCCIÓN}

La guayaba es un cultivo originario de América

\section{ABSTRACT}

In the present research, the optimization of the conservation of guava (Psidium guajava) in liquid silage using surface-response methodology was studied, depending on the variables: guava maturity stage, cachaça concentration and fermentation time. The variable response was the highest antioxidant activity equivalent to vitamin C (mg / $100 \mathrm{~g}$ ), during the production of guava liquid ensilage, determined by spectrophotometry. The values of the evaluated variables were: maturity stage (mature, pintón and verde), cachaza (250, 200 and $150 \mathrm{ml})$ and 15, 25 and 35 days of fermentation. The experimental design of Box-Behnken was used and a maximum concentration of total anthocyanins (ATa) of $3.72 \mathrm{mg} / 100 \mathrm{~g}$ was predicted and the ABTS (method to determine the antioxidant activity) was applied $133 \mathrm{mg} / 100 \mathrm{~g}$ of ECCEV (antioxidant activity Equivalent to vitamin C). The values of the variables were: mature fruits of guava, $250 \mathrm{~mL}$ of cachaça diluted in 1 liter of table water and 35 days of fermentation. To the results of applying the Box-Behnken design, the multiple regression analysis was applied to determine the coefficients of the polynomial equations, in order to obtain response surface lines for the determination of the antioxidant activity equivalent to vitamin C. All these calculations and statistical graphs were made in Statgraphics v. 5.1. With these optimum values guava liquid ensilage was elaborated, which was submitted to physicochemical analysis, spectrophotometric analysis and organoleptic evaluation. The tests gave satisfactory results for use in the dietary supplement of smaller animals.

\section{KEYWORDS}

Optimization $\cdot$ silage $\cdot$ liquid $\cdot$ guava

\footnotetext{
'Ingeniero Agrónomo. Docente de la UNTRM. Correo electrónico: vicente.castañeda@untrm.edu.pe

${ }^{2}$ Ingeniero Agroindustrial. Investigador del INNA y docente de la UNTRM. Correo electrónico: polito.huayama@untrm.edu.pe

${ }^{3}$ Licenciado en Administración. Docente de la UNTRM. Correo electrónico: jonathan.campos@untrm.edu.pe
} 
nal. A estos frutos, se los encuentra en cualquier parte del campo en forma silvestre, especialmente en las zonas tropicales o valles (Amin y Mukhrizah, 2006).

La guayaba ha tomado gran importancia comercial por su agradable aroma, el gran contenido de vitamina C, calorías, escasas proteínas y su reconocida propiedad de efecto laxante que la convierten en la fruta de mayor opción para la alimentación (Cristina, 2004).

Los desechos de frutas y hojas tienen un gran potencial forrajero, con un alto contenido en azúcares. El ensilaje es el mejor método para conservarlas, mezcladas con otros ingredientes para asegurar una buena fermentación y mejorar la calidad y la condición del ensilado (Stephen, 1999). Uno de los métodos de campo más usados en la preservación de productos y subproductos agrícolas, es el ensilaje. Este método consiste en conservar el producto sin tratamiento térmico, mediante el uso de la fermentación acidoláctica en estado semisólido provocada por las bacterias anaeróbicas que actúan sobre el sustrato disponible en el proceso (Mc Donald et al., 1981).

El ensilaje líquido es un proceso simple, económico y fácil de realizar. Se pueden llegar a conservar productos como la papa, la yuca y el apio en un medio acuoso y ácido generado por el crecimiento y multiplicación de los microorganismos y la interacción entre los azucares, almidones y los mismos microorganismos presentes en la cáscara de estos alimentos para posteriormente alimentar a los ovinos y caprinos (Maria et al., 2009).

La producción de guayaba en el distrito de Huambo, provincia de Rodríguez de Mendoza, región Amazonas, presenta una fuerte incidencia de larvas de la mosca de la fruta de género Anastrepha sp. que alcanza daños hasta del cien por ciento del fruto, dificulta el manejo adecuado de poscosecha, imposibilita el uso de la fruta para el proce- samiento agroindustrial y le resta significativamente el costo por $\mathrm{kg}$ S/ 0,20. El sobrante de la producción de guayaba infestada por la plaga dispersa por la zona, es utilizada de manera casera para la alimentación y cría de cerdos, vacas y aves de corral que encuentran en la guayaba aparentes ventajas en la producción de carne y huevos. Las gallinas que reciben guayaba en la alimentación que se les proporciona, ponen huevos con yemas visiblemente más amarillas, posiblemente por su elevado contenido de carotenos.

En esta investigación se realizó la optimización de la conservación de guayaba (Psidium guajava) en ensilado líquido mediante metodología superficie-respuesta, determinando las características fisicoquímicas, organolépticas y análisis espectrofotometrico para uso como alimento de animales menores (cuyes, conejos y gallinas).

\section{MATERIALES Y MÉTODOS \\ Materiales}

Se utilizó guayaba (Psidium guajava) que provino del distrito de Huambo. La cachaza fue proporcionada por centros de producción artesanal de panela. Además, se utilizó agua de mesa de la planta de producción Santa Bárbara. Finalmente, otro de los elementos utilizados fue el aceite vegetal, de un establecimiento comercial del distrito de Huambo.

\section{Preparación del ensilado líquido:}

El ensilado líquido de guayaba se elaboró en el laboratorio de la Estación Experimental de la Universidad Nacional Toribio Rodríguez de Mendoza, sede Rodríguez de Mendoza. Los frutos de guayaba se clasificaron y seleccionaron en base a sus características físicas (tamaño, forma y color), en tres estados de madurez fisiológica (verde, pintón y maduro) (Figura 1). Seguidamente se las lavó con agua a fin de eliminar algunas partículas extrañas que estén impregnadas en el fruto y se pesó para determinar el rendimiento.

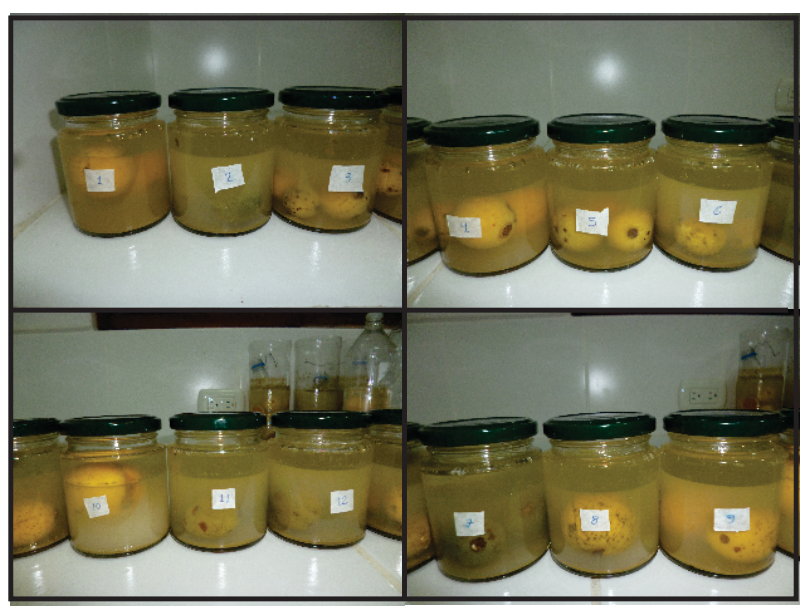

Figura 1. Preparación del ensilado de guayaba en el laboratorio 
Los frutos de guayaba seleccionados según el estado de madurez fisiológica fueron depositados en baldes que contenían agua de mesa con cachaza diluida en proporciones determinadas (relación 1 $\mathrm{kg}$ de fruta en 5 litros de dilusión). Se cubrió la solu- ción con aceite vegetal, con la finalidad de aislar del medio ambiente y se dejó fermentar por tiempos determinados, según los valores dados en la tabla 1, de acuerdo al diseño experimental de BoxBehnken para el proceso de fermentación (Tabla 5).

Tabla 1. Valor de las variables a evaluar con el diseño estadístico de Box-Behnken.

\begin{tabular}{lccc}
\hline Concentración & Estado de Madurez $\left({ }^{\circ}\right.$ Brix) A & Cachaza $(\mathbf{m L})$ B & Fermentación(días) C \\
\hline Alta (+) & Maduro (15) & 250 & 35 \\
Media (0) & Pintón (10) & 200 & 25 \\
Baja (-) & Verde (5) & 150 & 15 \\
\hline
\end{tabular}

\section{Acondicionamiento de los biorreactores:}

Los biorreactores fueron baldes de $8 \mathrm{~L}$ de capacidad, con iguales características y proporciones geométricas, donde se realizó el proceso de fermentación. Para realizar los experimentos se emplearon 15 baldes acondicionados .

Fase fermentativa. Proceso de obtención del ensilado líquido

En cada balde se colocó $5 \mathrm{~L}$ de agua de mesa que fue suplementada con cachaza, se agregó $1 \mathrm{~kg}$ de frutas de guayaba en sus diferentes estados de madurez fisiológica (verde, pintón y maduro) y se dejó fermentar durante 15, 25 y 35 días, de acuerdo a lo establecido en el diseño de BoxBehnken. Luego se homogenizó y se selló herméticamente con medio litro de aceite vegetal por cada experimento. Se colocó en un ambiente adecuado para dicho proceso. Esos experimentos contó con tres repeticiones y se reportó el promedio de los resultados.

\section{Fase post-fermentativa}

Después de cada período fermentativo se tomaron muestras para determinar los valores fisicoquímicos, espectrofotométricos y organolépticos producidos.

Optimización del proceso de fermentación

De las diversas variables de las cuales depende el proceso de fermentación, en la presente investigación, se consideró como las variables más importantes: la concentración de cachaza, estado de madurez de la guayaba y tiempo de fermentación. Los valores de estas variables fueron evaluados a partir del diseño de Box-Behnken. Los resultados se emplearon para dar valor agregado a la guayaba de la región Amazonas. Luego de la realización de todos los ensayos experimentales, y a partir de los datos espectrofotométricos (variable respuesta), se procedió a realizar el análisis estadístico para determinar los valores óptimos para la optimización de la conservación de la guayaba (Psidium guajava) en ensilado líquido mediante metodología superficie-respuesta

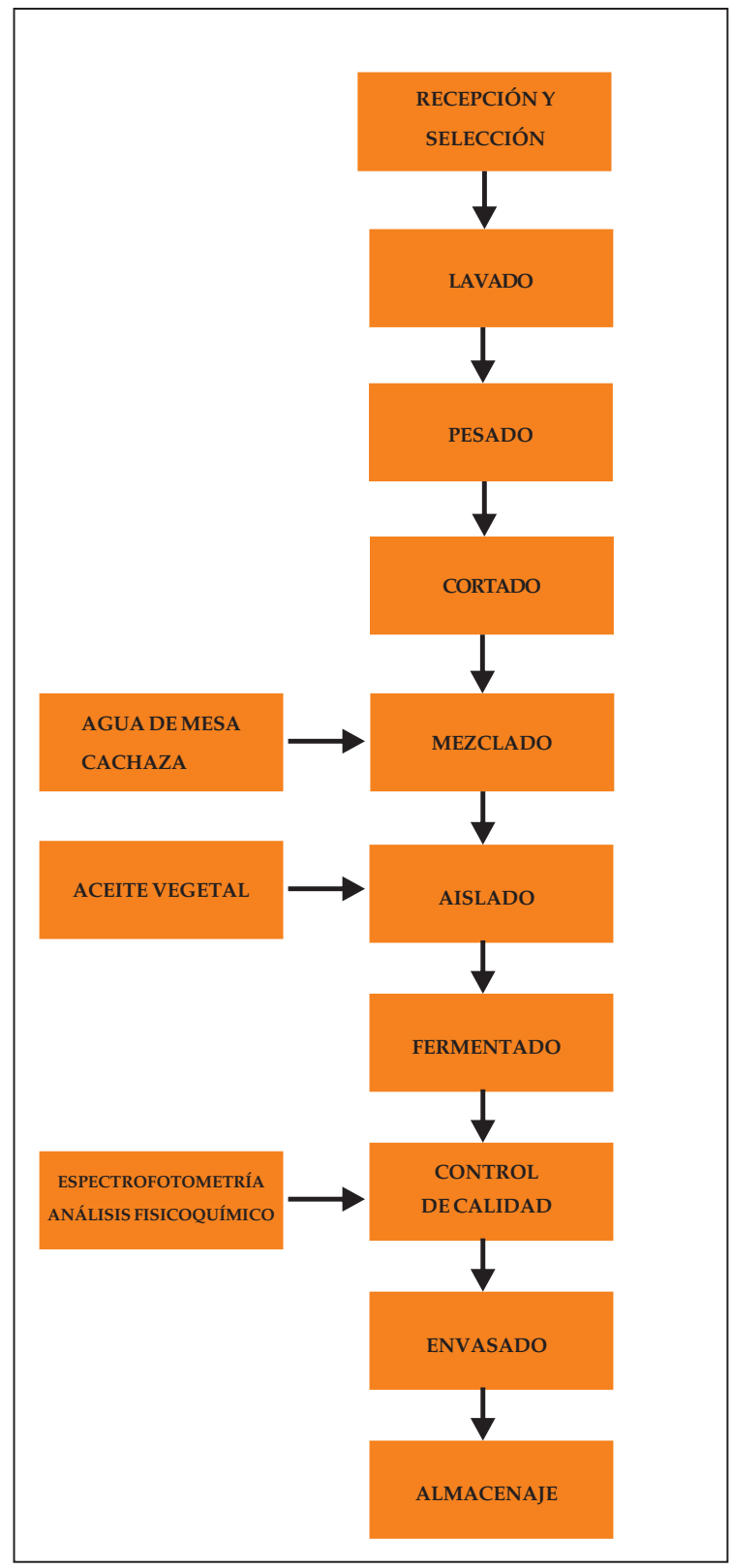

Figura 2. Diagrama de flujo del proceso de obtención de ensilado líquido de guayaba. 


\section{Análisis espectrofotométrico}

Las frutas fermentadas fueron extraídas según lo indicado en la tabla 5. Luego se colocaron en embalajes de $100 \mathrm{~g}$, conservadas bajo congelación a $-15^{\circ} \mathrm{C}$. Las muestras se prepararon de acuerdo con la indicación del fabricante, $100 \mathrm{~g}$ se disolvieron en $250 \mathrm{~mL}$ de agua Milli-Q, y se diluyeron con etanol y agua Milli-Q, según el método aplicado y luego se centrifugaron a $14000 \mathrm{rpm}$ durante 15 min. El registro de los espectros de absorción y las medidas de absorbancia, a longitud de onda fija, se llevaron a cabo con un Espectrofotómetro HP modelo 8452A (Cheadle Heath, Stockport Cheshire, UK).

Determinación de antocianos totales (AT) Método por diferencia de $\mathbf{p H}$.

Este método permite la estimación alternativa del contenido de antocianos totales. Se utilizaron dos sistemas tampón: ácido clorhídrico/cloruro de potasio de $\mathrm{pH} 1,0(0,025 \mathrm{M})$ y ácido acético/acetato sódico de pH 4,5 (0,4 M) A 0,2 mL de una muestra diluida (para conseguir una absorbancia en el rango de 0,100-1,200 a $510 \mathrm{~nm}$ ). Se añadieron $1,8 \mathrm{~mL}$ de la correspondiente disolución tampón y se midieron la absorbancia frente a un blanco a 510 y 700 nm. Se calculó la absorbancia final a partir de:

$$
\mathrm{A}=\left(\mathrm{A}_{\text {max.vis }}-\mathrm{A}_{700 \mathrm{~nm}}\right)_{\mathrm{pH} 1,0}-\left(\mathrm{A}_{\max \mathrm{vis}}-\mathrm{A}_{700 \mathrm{~nm}}\right)_{\mathrm{pH} 4,5}
$$

La concentración de pigmentos monoméricos en el extracto se expresó en cianidina-3-glucósido. Antocianos monoméricos $(\mathrm{mg} / 100 \mathrm{~g})=$

$$
\frac{\mathrm{A} \times \mathrm{PM} \times \mathrm{FD} \times 100}{(\varepsilon \times 1)}
$$

$\mathrm{A}=$ Absorbancia

$\mathrm{PM}=$ Peso molecular

$\mathrm{FD}=$ Factor de dilución

$\varepsilon=$ Absortividad molar

La concentración final de antocianos (mg/100 g) se calculó en base al volumen de extracto y peso de muestra. Se expresó en cianidina 3-glucósido (PM: 449,2 y $\varepsilon: 26900)$.

\section{Actividad antioxidante}

\section{Método ABTS.}

Según la metodología desarrollada por Re et al. (1999) y descrita por Kuskoski et al. (2005), el radical ABTS+ se obtiene después de la reacción de ABTS $(7 \mathrm{mM})$ con persulfato potásico $(2,45 \mathrm{mM}$, concentración final), incubado a temperatura ambiente $\left( \pm 25^{\circ} \mathrm{C}\right)$ y en la oscuridad durante $16 \mathrm{~h}$. Una vez formado el radical ABTS+ se diluye con etanol hasta obtener un valor de absorbancia, comprendido entre $0,70( \pm 0,1)$ a $754 \mathrm{~nm}$ (longitud de onda de máxima absorción). Las muestras filtradas (antocianos) se diluyen con etanol hasta que se produce una inhibición del $20 \%$ al $80 \%$, en comparación con la absorbancia del blanco, tras añadir $20 \mu \mathrm{L}$ de la muestra. A $980 \mu \mathrm{L}$ de dilución del radical ABTS+ generado se le determina la A754 a $30^{\circ} \mathrm{C}$, se añade $20 \mu \mathrm{L}$ de la muestra (dilución de antocianos) y se mide de nuevo la A754 pasado un minuto. La absorbancia se mide de forma continua transcurridos 7 minutos. El antioxidante sintético de referencia, Trolox, se ensaya a una concentración de 0-15 $\mu \mathrm{M}$ (concentración final) en etanol, en las mismas condiciones, lo que se hace también con ácido ascórbico (0-20 mg/100 mL). Los resultados se expresan en TEAC (actividad antioxidante equivalente a Trolox) y en VCEAC (actividad antioxidante equivalente a vitamina $\mathrm{C}$ ), en este último caso por tratarse de alimentos.

\section{Análisis estadístico}

El nivel óptimo de cada variable independiente se determinó usando las técnicas de Optimización de Respuesta Superficial (McDaniel et al., 1976), las cuales se aplicaron a las 3 variables independientes y sus relaciones se ajustaron al siguiente modelo polinomial cuadrático.

$$
\mathrm{Z}=\mathrm{b}_{0}+\mathrm{b}_{1} \mathrm{~A}+\mathrm{b}_{2} \mathrm{~B}+\mathrm{b}_{3} \mathrm{C}+\mathrm{b}_{4} \mathrm{AB}+\mathrm{b}_{5} \mathrm{AC}+\mathrm{b}_{6} \mathrm{BC}+\mathrm{b}_{7} \mathrm{~A}^{2}+\mathrm{b}_{8} \mathrm{~B}^{2}+\mathrm{b}_{9} \mathrm{C}^{2}
$$

Dónde:

$\mathrm{Z}=$ Variable dependiente (VCEAC)

A, B, C = Variables independientes

$\mathrm{b}_{0}=$ Coeficiente de regresión en el punto central

$\mathrm{b}_{1}, \mathrm{~b}_{2}, \mathrm{~b}_{3}=$ Coeficientes lineales

$\mathrm{b}_{4}, \mathrm{~b}_{5}, \mathrm{~b}_{6}=$ Coeficientes de interacción de segundo orden

$\mathrm{b}_{7}, \mathrm{~b}_{8}, \mathrm{~b}_{9}=$ Coeficientes cuadráticos.

Para la identificación de un valor óptimo fue necesario estimar la curvatura. Para ello, cada variable fue ensayada en quince niveles, mediante el diseño de Box-Behnken que es un diseño factorial fraccional (Daniel, 1976).

Los coeficientes del modelo polinomial fueron calculados usando las técnicas de regresión múltiple y luego se escribió las ecuaciones XY polinomiales y fueron usadas para generar respuestas y líneas de contorno para las diferentes combinaciones de los niveles de las variables. Todos los cálculos y gráficos estadísticos fueron realizados con el software Statgraphics v. 5.1.

\section{RESULTADOS}

En la tabla 3, se presenta los resultados de las características fisicoquímicas de la pulpa de guayaba en sus tres estados de madurez (madura, pintón y verde). La clasificación se realizó visualmente. 
Tabla 2. Características fisicoquímicas de la pulpa de guayaba

\begin{tabular}{lccccc}
\hline Características & $\mathbf{p H}$ & ${ }^{\circ}$ Brix & \% Acidez & (IM) & Humedad \% \\
\hline Madura & 4,23 & 9,63 & $0,75 \pm 0,07$ & 13,62 & $83,4 \pm 0,1$ \\
Pintón & 4,37 & 9,25 & $0,72 \pm 0,07$ & 11,54 & $82,7 \pm 0,1$ \\
verde & 4,54 & 8,31 & $0,69 \pm 0,07$ & 9,27 & $82,1 \pm 0,1$ \\
\hline
\end{tabular}

*Expresado en ácido cítrico, (IM) Índice de Madurez

En la tabla 3, se presenta los resultados de las características fisicoquímicas de la cachaza que se utilizó como medio edulcorante para el ensilado líquido de guayaba.

Tabla 3. Características fisicoquímicas de cachaza

\begin{tabular}{ccc}
\hline Características & $\mathbf{p H}$ & ${ }^{\circ}$ Brix \\
\hline Cachaza & 4,5 & 32 \\
\hline
\end{tabular}

En la tabla 4, se presenta los resultados del análisis espectrofotométrico realizado a la pulpa de guayaba, antes del proceso de ensilado líquido.
Tabla 4. Análisis espectrofotométrico de la pulpa de guayaba fresca

\begin{tabular}{ccc} 
& \multicolumn{2}{c}{ VCEAC(mg/100 g) } \\
\cline { 2 - 3 } Características & ATa & ABTS (1 $\mathbf{~ m i n )}$ \\
\hline Madura & 2,96 & 120 \\
Pintón & 2,60 & 118 \\
verde & 1,42 & 96 \\
\hline
\end{tabular}

$\mathrm{AT}^{\mathrm{a}}=$ Antocianos totales

ABTS=Método para determinar la actividad antioxidante VCEAC $=$ Actividad antioxidante equivalente a vitamina $\mathrm{C}$

De los resultados del análisis espectrofotométrico VCEAC (actividad antioxidante equivalente a vitamina C) de la tabla 5, los valores de las variables independientes del ensayo 14 son los que evidenciaron la mayor concentración de vitamina $\mathrm{C}$.

Tabla 6. Resultado de análisis espectrofotométrico VCEAC

\begin{tabular}{cccccc}
\hline \multirow{2}{*}{$\begin{array}{c}\text { Número } \\
\text { experimento }\end{array}$} & A & B & C & AT $^{\mathbf{a}}$ & ABTS (1 min.) \\
\cline { 2 - 6 } & 15 & 250 & 25 & 3,42 & 127 \\
2 & 5 & 250 & 35 & 2,19 & 112 \\
3 & 10 & 150 & 25 & 2,51 & 115 \\
4 & 15 & 150 & 15 & 3,25 & 121 \\
5 & 10 & 250 & 25 & 2,65 & 119 \\
6 & 10 & 250 & 15 & 2,26 & 114 \\
7 & 5 & 150 & 35 & 2,16 & 109 \\
8 & 10 & 150 & 15 & 2,21 & 113 \\
9 & 15 & 200 & 35 & 3,62 & 131 \\
10 & 15 & 200 & 25 & 3,36 & 123 \\
11 & 5 & 200 & 35 & 2,17 & 111 \\
12 & 5 & 200 & 15 & 1,96 & 98 \\
13 & 10 & 200 & 25 & 2,62 & 118 \\
14 & 15 & 250 & 35 & 3,72 & 133 \\
15 & 5 & 150 & 15 & 1,88 & 104 \\
\hline
\end{tabular}

Maduro 15, pintón 10, verde 5 (mongomeri)

$\mathrm{At}^{\mathrm{a}}=$ Antocianos totales

ABTS=Método para determinar la actividad antioxidante

VCEAC=Actividad antioxidante equivalente a vitamina $\mathrm{C}$

Para el análisis de Antocianos totales (AT), se determinó el análisis de varianza y el coeficiente de determinación $\mathrm{R}^{2}=97,60 \%$, indicando que se ha conseguido explicar el $97 \%$ de la variabilidad total de la variable dependiente. La ecuación del modelo ajustado, para lograr el valor óptimo, es: $\mathrm{AT}^{\mathrm{a}}=1,61164-0,0650355 *$ Madurez $0,000944941 *$ Cachaza $+0,0320929 *$ Fermentación $+0,00931781$ * Madurez $^{\wedge} 2-0,0000936262$ * Madurez * Cachaza $+0,00125815$ * Madurez * Fermentación + 0,00000364753* Cachaza^2 +
0,0000324397 * Cachaza * Fermentación 0,000633175*Fermentación^2

Respuesta Optimizada

Meta: maximizar $\mathrm{AT}^{\mathrm{a}}$

Valor Óptimo $=3,66523$

Tabla 7. Valores óptimos de las variables independientes

\begin{tabular}{lccc}
\hline \multicolumn{1}{c}{$\begin{array}{c}\text { Variable } \\
\text { independiente }\end{array}$} & Inferior & Mayor & Óptimo \\
\hline Madurez & 5,0 & 15,0 & 15,0 \\
Cachaza & 150,0 & 250,0 & 250,0 \\
Fermentación & 15,0 & 35,0 & 35,0 \\
\hline
\end{tabular}


Se observa que a los 35 días (Figura 3) se logró la máxima concentración de $\mathrm{AT}^{\mathrm{a}}$ que alcanza a 3,7, con ensilado líquido suplementado con cachaza $250 \mathrm{~mL}$ y con frutos de guayaba madura.

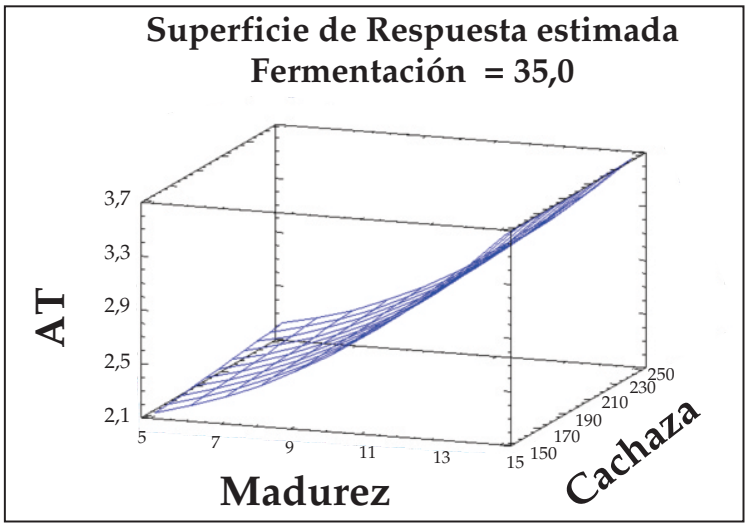

Figura 3. Superficie de respuesta de $\mathrm{AT}^{\mathrm{a}}$ en función de la madurez-cachaza, tiempo de ensilado (35 días). Máximo3,66523, de AT

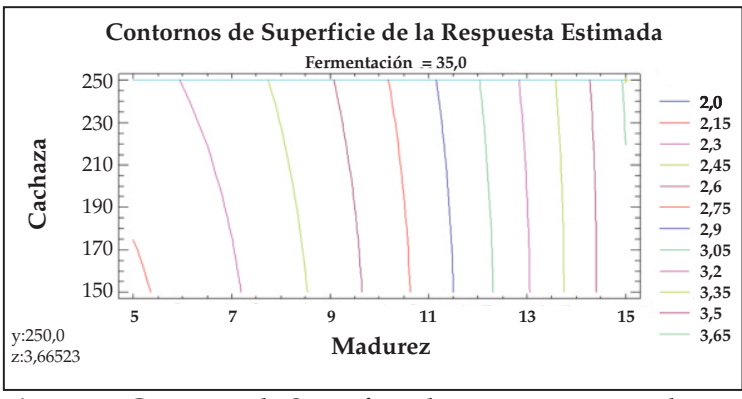

Figura 4. Contorno de Superficie de respuesta estimada que contrasta la figura 2 y los niveles Máximos 3,66523 de $\mathrm{AT}^{\mathrm{a}}$ alcanzados.

Para el análisis del método aplicado para determinar la actividad antioxidante (ABTS), se determinó el análisis de varianza y el coeficiente de determinación $\mathrm{R}^{2}=96,35 \%$, indicando que se ha conseguido explicar el $96 \%$ de la variabilidad total de la variable dependiente. La ecuación del modelo ajustado, para lograr el valor óptimo, es: ABTS $=104,958+3,62359 *$ Madurez $-0,142241$ * Cachaza $-0.833737 *$ Fermentación- 0,126227* Madurez^2 + 0,00625695* Madurez * Cachaza $0,0129797 *$ Madurez*Fermentación + 0,000054668* Cachaza^2 $+0,00329913 *$ Cachaza *Fermentación $+0,015581$ * Fermentación^ ${ }^{\wedge}$

Respuesta Optimizada

Meta: maximizar ABTS

Valor Óptimo = 134,19

Se observa que a los 35 días (Figura 4) se logró la máxima concentración de ABTS que alcanzó a 134 con ensilado líquido suplementado con cachaza $250 \mathrm{~mL}$ y con frutos de guayaba madura.

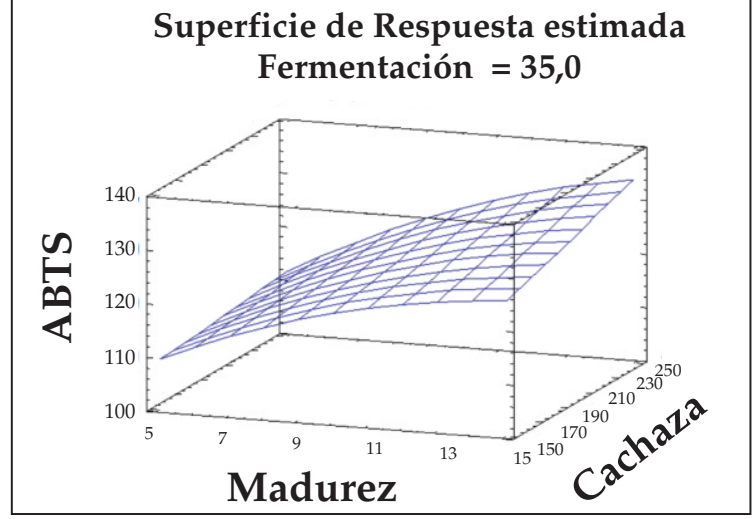

Figura 5. Superficie de respuesta de ABTS en función de la madurez-cachaza, tiempo de ensilado (35 días), Máximo 134,19 ABTS

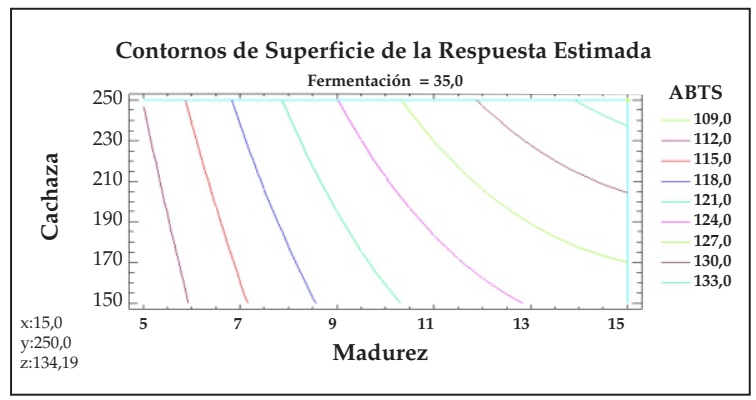

Figura 6. Contorno de Superficie de respuesta estimada que contrasta la figura 4 y los niveles Máximo 134,19 ABTS alcanzado.

En las figuras 7 y 8 , se aprecia la disminución del $\mathrm{pH}$ y el incremento de $\operatorname{los}^{\circ}$ Brix. En el transcurso de los días de ensilado, el tratamiento 14 tuvo mejor respuesta.

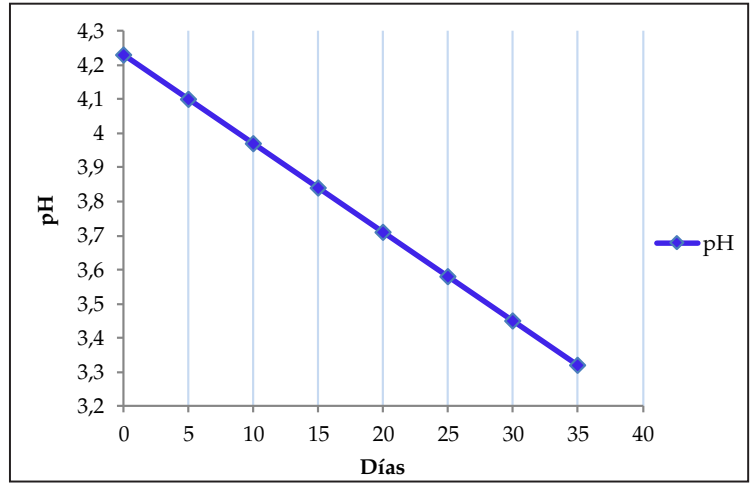

Figura 7. Variación del $\mathrm{pH}$ con respecto al tiempo durante el ensilado líquido 


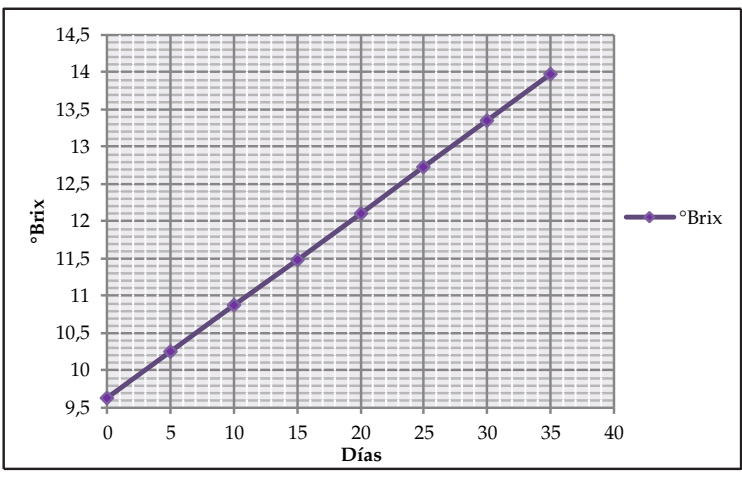

Figura 8. Variación de $\operatorname{los}^{\circ}$ Brix con respecto al tiempo, durante el ensilado líquido

En la figura 9, se aprecia la aceptación del ensilado líquido de guayaba del experimento $\mathrm{N}^{\circ} 14$. En cuanto al color (Me gusta moderadamente), Sabor (Me gusta ligeramente), Olor (Me gusta mucho), aspecto (Me gusta moderadamente).

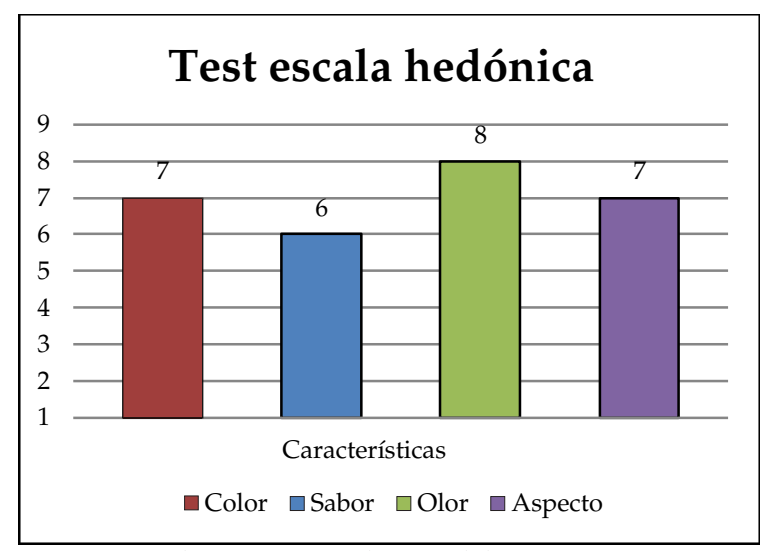

Figura 9. Evaluación organoléptica del experimento 14

\section{DISCUSIÓN}

Para el silaje de papa, se analizaron las características organolépticas (olor, color y consistencia), químicas y nutricionales. Estos resultados mostraron que los ensilados sin aditivo y con aditivos presentan características diferentes. Los primeros presentaron características organolépticas malas y una descomposición muy avanzada a los siete días. De los ensilados con aditivos solo uno mantuvo buenas características organolépticas, químicas y nutricionales, mientras que el otro ensilado presenta descomposición al día 15 de su confección (Bodega, 2013). En cambio, en el ensilado líquido de frutos de guayaba fermentado con cachaza diluida en agua de mesa y a los 35 días de fermentación se obtuvieron frutos de guayaba con buenas características organolépticas y aptas para uso en la alimentación de animales menores.

Para Guzmán (2010), los cambios en las características químicas de ensilados de mango, según épocas de cosecha pudieron, estar influidos, posi- blemente, por la naturaleza de las frutas utilizadas, que mostraron ciertas diferencias según el mes en que se cosecharon. La temperatura ambiental podría ser un factor que modifica el proceso fermentativo (McCullough, 1975; McDonald et al., 1991). Aún así, en esta investigación no hubo evidencias de cambios notables en la temperatura ambiental entre días. Se sabe que existen factores de manejo agronómico que se pueden reflejar en el rendimiento y las características de las frutas (Quijada et al., 2009; Yeshitela et al., 2005), pero este aspecto no fue materia de la presente investigación.

En relación a los aditivos, Bolsen et al. (1996) refiere que los aditivos, que se incluyen en la preparación de los silos, se caracterizan por una alta concentración de carbohidratos fermentables, baja capacidad amortiguadora, materia seca relativamente baja, entre 20 y $30 \%$, y bacterias lácticas adecuadas. Es interesante señalar que se utilizaron residuos de mango como aditivo para materiales a ensilar más convencionales, con el propósito de alcanzar índices adecuados en los silos (Filho et al., 2010; Rêgo et al., 2010) y viceversa. Los residuos con diferentes aditivos (Filho et al., 2006). Este último tipo de ensilado ha sido de los preparados en estudios realizados en México (Aguilera et al., 1997; Scotillo, 1984). En la presente investigación no se utilizaron aditivos porque el proceso de fermetación se realizó con bacterias presentes en el fruto de guayaba.

Entre los métodos químicos utilizados para determinar la capacidad antioxidante (captación de radicales libres), el radical ABTS+ es uno de los más rápidos porque proporciona resultados reproducibles y coherentes. Además, el ABTS presenta importantes ventajas ya que muestra varios resultados máximos de absorción y una buena solubilidad. Además, permite el ensayo de compuestos tanto de naturaleza lipofílica como hidrofílica. El tiempo de un minuto, para el método ABTS, puede ser suficiente para medidas de pulpas de frutos. En la presente investigación, para determinar la actividad antioxidante equivalente a vitamina $C$, se utilizó el método ABTS (1 min) y se obtuvieron resultados satisfactorios para pulpa de guayaba fresca 2,96 mg/100 g de Antocianos totales (AT $)$ y $120 \mathrm{mg} / 100 \mathrm{~g}$ de actividad antioxidante (ABTS), resultados similares a lo reportado por Kuskoski et al. (2005) para pulpa congelada de guayaba 2,7 $\mathrm{mg} / 100 \mathrm{~g}$ de Antocianos totales $\left(\mathrm{AT}^{\mathrm{a}}\right)$ y 120 $\mathrm{mg} / 100 \mathrm{~g}$ de actividad antioxidante (ABTS) y para frutos de guayaba ensilada $3,72 \mathrm{mg} / 100 \mathrm{~g}$ de Antocianos totales $\left(\mathrm{AT}^{\mathrm{a}}\right)$ y $133 \mathrm{mg} / 100 \mathrm{~g}$ de actividad antioxidante (ABTS). Este incremento se debe al 
índice de madurez $(13,62)$ y a la variación de los ${ }^{\circ}$ Brix de 9,63 a 13,97.

Asimismo, en las condiciones de operación para elaborar el ensilado líquido, donde se incluyen los valores óptimos de las variables independientes para obtener la mayor actividad antioxidante equivalente a vitamina $\mathrm{C}$ (experimento 14), el pH inicial del ensilado líquido fue de 4,23 dentro del rango de 3,40-5,0 que favorece el crecimiento óptimo de levaduras e inhiben el desarrollo de bacterias. Además, la evaluación organoléptica del ensilado líquido de guayaba dio resultados aceptables para su uso (Figura 9), y convierte este proceso en una alternativa adecuada para dar valor agregado a la guayaba y disminuir el desperdicio de esta fruta en campo.

Al hacer el seguimiento a la fermentación durante los 35 días, se midió el $\mathrm{pH}$ y el ${ }^{\circ}$ Brix, del ensilado líquido de guayaba, se encontraron que sus valores de $\mathrm{pH}$ desciendieron casi linealmente lo cual es característico en las fermentaciones. Simultáneamente, los valores de ${ }^{\circ}$ Brix ascendieron casi linealmente. Esto se debe a que los frutos continuaron con su proceso de maduración. Al final del proceso de fermentación del ensilado líquido, se realizó el análisis espectrofotométrico y organoléptico. Se obtuvo, a partir de estos, una buena aceptación para el uso en el suplemento alimenticio de animales menores.

Como estrategia alimentaria, el ensilaje de frutos como banano y follajes nativos es una alternativa importante en la alimentación de animales menores, en especial cuando existen limitaciones de productividad natural por factores estacionales (Chedly y Lee, 2000). Esto queda demostrado en el trabajo de investigación donde se muestra el aprovechamiento de la guayaba ensilada.

\section{CONCLUSIONES}

Se ha realizado el ensilado líquido a partir de frutos de guayaba, con resultados satisfactorios en la evaluación espectrofotométrica, fisicoquímica y organoléptica.

La secuencia de trabajo para elaborar ensilado líquido de guayaba se inició con la recepción, selección, lavado, pesado, de frutas de guayaba maduras, fermentada por 35 días en mezcla de cachaza diluida en agua de mesa, cubierta con aceite vegetal como aislante del medio ambiente y almacenada con la finalidad de utilizarlo como suplementado alimenticio de animales menores. Este proceso biotecnológico es adecuado para dar valor agregado a la guayaba y prolongar su vida útil como suplemento en la alimentación de animales menores.
Los valores óptimos encontrados de las variables independientes son: Frutos de guayaba en estado de madurez $=$ Maduro $\left({ }^{\circ}\right.$ Brix $\left.=9,63\right)$, Cachaza $=$ $250 \mathrm{ml}$, Tiempo de fermentación $=35$ días.

Las condiciones de operación finales para el ensilado líquido fermentado por 35 días en $250 \mathrm{~mL}$. de cachaza diluida en un litro de agua de mesa a temperatura ambiente de guayaba fueron: frutos maduros de guayaba de $\mathrm{pH} 4,23{ }^{\circ} \mathrm{Brix} 9,63$, porcentaje de Acidez 0,75 $\pm 0,07$; Índice de Madurez 13,62; Porcentaje de humedad 83,4 $\pm 0,1$.

\section{REFERENCIAS BIBLIOGRÁFICAS}

Aguilera, A., Pérez-Gil, F., Grande, D., De la Cruz, I., \& Juárez, J. (1997). Digestibility and fermentative characteristics of mango, lemon and corn stover silages with or without addition of molasses and urea. Small Ruminant Research, 26(1), 87-91.

Amin, I., \& Mukhrizah, O. (2006). Antioxidant capacity of methanolic and water extracts prepared from food-processing by-products. Journal of the Science of Food and Agriculture, 86(5), 778-784.

Bodega, J. L. (2013). Silaje de papa para alimentación animal. Producir XXI. a. 18, (224).

Bolsen, K. K., Ashbell, G., \& Weinberg, Z. G. (1996). Silage fermentation and silage additives-Review. Asian-Australasian Journal of Animal Sciences, 9(5), 483-494.

Chedly, K., \& Lee, S. (2000). Silage from byproducts for smallholders. FAO Plant Production And Protection Papers, 85-96.

Cristina, R. (2006). Prueba de concepto para los nuevos productos a base de guayaba desarrollados por Corpoica en la estación experimental Cimpa: sabajón, dulce de leche y gomitas ácidas (informe final del contrato de prestación de servicios). Bogotá, IICA, Barbosa, Santander, Corpoica EE Cimpa.

Filho, C.C.C.C., Filho, J.C.S., Junior, A.P.N., Freitas, R.T.F., Souza, R.M. \& Nunes, J.A.R. (2006). Qualidade da silagem de residuo de manga com diferentes aditivos. Ciencia e Agrotectenia (Lavras) 32:1537

Filho, C.C.C.C., Filho, J.C.S., Junior, A.P.N., Souza, R.M.S., Nunes, J.A.R. \& Coelho, J.V. (2010). Fraçoes fibrosas da silagem de residuo de manga com aditivos. Ciencia $e$ Agrotecnia (Lavras) 34:751

Guzmán, O. (2010). Estudio de conservación de desechos de mango (Mangifera indica L.) para la alimentación de ovinos en el Estado de Nayarit (Disertación doctoral, tesis de maestría). Universidad Autónoma de Nayarit. 
Tepic. México.

Kuskoski, E. M., Asuero, A. G., Troncoso, A. M., Mancini-Filho, J., \& Fett, R. (2005). Aplicación de diversos métodos químicos para determinar actividad antioxidante en pulpa de frutos. Food Science and Technology (Campinas), 25(4), 726-732.

Maria V. \& Estevan M. (2009), Elaboración de ensilado líquido a base de yuca, papa y apio como una alternativa de alimentación en la época de verano para ovino y caprino del centro de investigación Pecuario Guatiguara, Bucaramanga, Colombia.

McDaniel, L. E., Bailey, E. G., Ethiraj, S., \& Andrews, H. P. (1976). Application of response surface optimization techniques to polyene macrolide fermentation studies in shake flasks. Dev Ind Microbiol, 17, 91-98

McDonald, P., Edwards, R. A., \& Greenhalgh, J. F. D. (1981). Animal nutrition. Editorial. Acribia. España.

McCullough, M. E. (1975). Nuevas tendencias en ensilaje de forrajes. Rev. Mundial de Zootecnia, 15, 44.

McDonald, P., Henderson, A. R., \& Heron, S. J. E. (1991). The Biochemistry of Silage. Marlow: Chalcombe. 340 pp.

Quijada, O., Villacorta, B. H., González, R., Casanova, Á., \& Camacho, R. (2009). Influencia de la poda y de la aplicación de nitrato potásico y tiosulfato potásico sobre el mango en Maracaibo, Venezuela. II. Producción e índices de eficiencia productiva. Agronomía Tropical, 59(3), 289296.

Re, R., Pellegrini, N., Proteggente, A., Pannala, A., Yang, M., \& Rice-Evans, C. (1999). Antioxidant activity applying an improved ABTS radical cation decolorization assay. Free radical biology and medicine, 26(9), 1231-1237.

Rêgo, M. M. T., Neiva, J. N. M., Rêgo, A. C. D., Cândido, M. J. D., Carneiro, M. S. D. S., \& Lôbo, R. N. B. (2010). Chemical and bromatological characteristics of elephant grass silages containing a mango byproduct. Revista Brasileira de Zootecnia, 39(1), 81-87.

Scotillo, F. V. G. (1984). Factibilidad, evaluación y digestibilidad in vitro del ensilaje de mango con la adición de rastrojo de maíz. (Tesis de pregrado). Instituto Tecnológico y de Estudios Superiores de Monterrey. Monterrey, México.

Stephen, L. (1999) Uso del ensilaje en el trópico privilegiando opciones para pequeños campesinos, Memorias de la Conferencia Electrónica de la FAO sobre el Ensilaje en los Trópicos.

Yeshitela, T., Robbertse, P. J., \& Stassen, P. J. C. (2005). Potassium nitrate and urea sprays affect flowering and yields of 'Tommy Atkins'(Mangifera indica) mango in Ethiopia. South African Journal of Plant and Soil, $22(1), 28-32$. 